\title{
Das Haus als Spiegel tieferer Vorgänge im Schweizerspiegel von Meinrad Inglin
}

doi.org/10.14232/jp.agi.2022.3.1

BETREUERIN: DR. CSILLA MiHÁLY

„Ein neues Haus, ein neuer Mensch.”

Johann Wolfgang von Goethe

\section{Einleitung}

Eine bekannte ungarische Schriftstellerin, Magda Szabó denkt, dass in einem literarischen Werk das Zuhause von dem Protagonisten untrennbar ist. (vgl. Aczél 1997, S. 141) Mit diesem Gedanken im Hinterkopf las ich den Schweizerspiegel, den berühmtesten Roman des schweizerischen Schriftstellers, Meinrad Inglins.

Der Schweizerspiegel lässt sich als der schweizerische Krieg und Frieden und der schweizerische Buddenbrooks-Roman ansehen. Er ist ein Familienroman, der die ganze Gesellschaft durch die einzelnen Protagonisten veranschaulicht. Es gibt zahlreiche Aspekte, die man in diesem Roman untersuchen kann. Ich habe mich für die Untersuchung des Raumkonzepts im Roman entschieden. Diese Entscheidung habe ich einerseits darum getroffen, weil das Haus als Motiv eine leitende Rolle in dem Roman spielt; andererseits, weil die Raumforschung eine relativ neue Disziplin der Literaturforschung ist. Mein Ziel ist mit meiner Arbeit die Aufmerksamkeit der Literaturforschung auf neue, bisher vielleicht im Hintergrund stehende Gebiete zu lenken. 
Meine Arbeit besteht aus zwei größeren Teilen. Im ersten theoretischen Teil schreibe ich über den Autor, weil sein Name unter den Literaturforschern wenig bekannt ist, und fasse den Inhalt des Romans kurz zusammen, damit die Interpretation im zweiten Teil verständlich ist. Als nächstes stelle ich die Raumforschung mit den für meine Arbeit relevanten Konzepten vor, um den wissenschaftlichen Hintergrund meiner Arbeit $\mathrm{zu}$ zeigen. Zuerst schreibe ich über die Raumforschung allgemein, dann über die Aspekte, die bei der Analyse des Romans wichtig sind. Am Ende des theoretischen Teils steht die ausführliche Beschreibung meiner Hypothese und der verwendeten Methode. Im zweiten Teil ist die Analyse des Hausmotivs im Roman zu finden. In der anschließenden Zusammenfassung möchte ich meinen Gedankengang und die wichtigsten Aussagen noch einmal zusammenfassen.

\section{Meinrad Inglin und der Schweizerspiegel}

Meinrad Inglin ist am 28. Juli 1893 in Schwyz als der ältere von zwei Söhnen geboren. (vgl. Inglin/Hangarten 1992, S. 15) Sein Vater, der Uhrmacher war, betrieb sein Geschäft im Erdgeschoss des eigenen Hauses und war daneben noch Goldschmied, Musikant, Jäger, Bergsteiger und Offizier. Mütterlicherseits war Inglin ein Urenkel von einem Regierungs- und Nationalrat und Hotelier (vgl. Inglin/Schoeck-Grüebler 2005, S. 18). Im Folgenden beschreibe ich sein Leben anhand seiner Selbstbiographie (vgl. Inglin/Hangarten 1992, S. 15-16). Mit dreizehn verlor er seinen Vater, mit sechzehn seine Mutter. Schon mit vierzehn beschloss er Schriftsteller zu werden, aber seine Erzieher haben daran festgehalten, er müsse einen rechten bürgerlichen Beruf erlernen. Er fing mehrere Lehren und später ein Studium in Bern an. Das Studium musste er unter- 
brechen und schließlich abbrechen, um Militärdienst zu leisten, und um seinen Unterhalt zu verdienen. Er hat eine Zeit lang in einer Redaktion in Bern als Volontär gearbeitet. Sein erstes Buch, Die Welt in Ingoldau, erschien 1922 und von dem an arbeitete er als Schriftsteller (vgl. Inglin/Hangarten 1992, S. 17). Er hat zahlreiche Bücher geschrieben, davon ist der Schweizerspiegel das größte und bekannteste Werk (vgl. Inglin/Hangarten 1992, S. 18). 1948 bekam er den Großen Schillerpreis der Schweizerischen Schillerstiftung (vgl. Inglin/Hangarten 1992, S. 15). Er starb am 4. Dezember 1971 in Schwyz (vgl. Inglin/Schoeck-Grüebler 2005, S. 157).

In einer repräsentativen Biographie und in dem wissenschaftlichen Schrifttum wird Inglin der Status "Klassiker der Moderne" der Schweiz gegeben (vgl. Rusterholz/Solbach 2007, S. 205). Seine Präsenz in dem Kanon liegt, sagt Rusterholz, einerseits in dem Umfang seines Lebenswerks, andererseits in der Repräsentativität seines Werkes (vgl. Rusterholz/Solbach 2007, S. 206). Dass er literarisch anerkannt war, war teilweise auch der freundschaftlichen Förderung Emil Steigers zu danken, stellt Hangartner fest (vgl. Inglin/Hangarten 1992, S. 20).

Der Roman Schweizerspiegel erschien 1938 im Geiste der Landesverteidigung. Inglins Stellungnahme in der politischen Verwirrung vor dem Zweiten Weltkrieg zeigt sein Brief an Bettina Zweifel vom 1. August 1937 sehr eindeutig. „Das Vaterland braucht vielleicht meinen Roman so gut wie die Bundesfeier." - schreibt Inglin. (Zitiert nach Matt 1976, S. 170) Die geistige Landesverteidigung war ein kulturpolitisches Programm, das die nationale Selbstbestimmung und Konsolidierung förderte (vgl. Egger 2004, S. 195). Der Schweizerspiegel schildert sowohl die gesellschaftlichen als auch die individuellen Veränderungsprozesse um die Jahrhundertwende und während des Ersten Weltkrieges. 
In dem Roman wird ein umfassendes Panorama von der damaligen Gesellschaft zwischen den Jahren 1912 und 1919 gezeigt (vgl. Rusterholz/Solbach 2007, S. 206), wo jede Gestalt für eine ganze Partei steht (vgl. Matt 2014, S. 878). Der Roman wurde aus bürgerlichem Standpunkt geschrieben und stellt die Krise des Liberalismus der Vätergeneration durch die Lebensgeschichte der vier Kinder von Amman vor. Inglin malt ein überzeugendes Bild historischer Ereignisse und Mentalitäten, dadurch, dass er die politischen und ideologischen Überzeugungen in der Handlung integriert (vgl. Rusterholz/Solbach 2007, S. 217). Durch das Leben der Familie des Nationalrats Amman werden die widerstrebenden politischen und kulturellen Denkweisen vorgestellt. Der Roman zeigt die Gefahren und Schwächen der verschiedenen ideologischen Positionen, bietet aber keine Lösung (vgl. Rusterholz/Solbach 2007, S. 206).

Wie Rusterholz und Solbach in ihrer Schweizer Literaturgeschichte feststellen, denke ich auch, dass durch die reine Wiedergabe der Handlung die künstlerische Seite des Romans teilweise verloren geht (vgl. Rusterholz/Solbach 2007, S. 217). Um die anschließende Interpretation nachvollziehen zu können, scheint es doch wichtig, die Handlung des Romans kurz darzulegen.

Im Mittelpunkt des Romans steht die angesehene Familie des Nationalrats und Obersten Alfred Ammann. Alfred Amman verfügt über die besten Beziehungen zu bedeutenden Politikern und Militärs und repräsentiert das gehobene Zürcher Bürgertum. Die Geschichte beginnt 1912 bei einem Manöver in der Nähe von Zürich. Da der deutsche Kaiser, Wilhelm II. die Schweiz besucht, ist das ganze Volk aufgeregt. An der allgemeinen Aufregung nimmt auch die Familie Amman teil. Der Vater, Alfred Amman und seine drei Söhne, Severin, Paul und Fred sind auch anwesend als der Kaiser das Manöver anschaut. 
Aber schon am Anfang der Geschichte ist es bemerkbar, wie sich die Meinungen der Geschwister in Bezug auf den Besuch des Kaisers spalten. Severin, der älteste Sohn Alfred Ammans ist sehr begeistert von dem kaiserlichen Besuch. Er ist Rechtsextremist und der Redakteur des Ostschweizers, einer deutschfreundlichen Zeitung. Seine germanophilen Gefühle werden mit der Zeit immer extremer. Paul, der mittlere Sohn findet die Aufregung um den Kaiser lächerlich und geht in kurzer Zeit nach Hause. Er behält seinen skeptischen Standpunkt auch in den folgenden Jahren und ist eher mit der linken Politik einverstanden. Er nimmt an den von den Sozialisten organisierten Ereignissen teil und er ist überhaupt davon überzeugt, dass der Sozialismus die Lösung für die sozialen Probleme ist. Fred, der kleinste Sohn weiß in dieser Situation nicht, wem er folgen sollte und er versteht die radikalen Gedanken seiner Brüder oft nicht. Aber wenn er zwischen den zwei Brüdern eine Wahl treffen muss, folgt er eher Paul. Bis zum Ende der Geschichte interessiert er sich wenig für die Politik. Er erfüllt seine soldatische Pflicht, er ist auch zu gewissen Maßen begeistert davon, aber nicht genug um Soldat zu werden. Lange weiß er auch nicht, was er studieren sollte, bis er am Ende herausfindet, dass er am liebsten Landwirt wäre.

In den folgenden zwei Jahren wird der Ausbruch eines Kriegs immer wahrscheinlicher. Obwohl die Schweizer Elite es wahrnimmt, kann sie es trotzdem nicht glauben. 1914 findet ein großes Schützenfest statt, wo es noch einmal zu beobachten ist, wie das alte System und die veralteten Einstellungen untergehen. Der Kriegsbeginn wurde meistens begeistert empfangen. In den darauffolgenden Szenen stellt Inglin eine eigentümliche schweizerische Erfahrung dar: die Mobilisierung ohne wirklichen Einsatz. Die Soldaten sind zwar kampf- und todesbereit, aber der Feind überschritt die Grenze nicht, die Soldaten dürfen ihren Mut nicht beweisen. Mit der Zeit entwickeln sich 
Frust und Hass auf den Krieg. Dieses Gefühl wurde dank den unzähligen Verwundeten- und Gefangenentransporten, die die Schweiz durchqueren, immer stärker. Auch der Konflikt zwischen den germanophilen Deutsch- und frankophilen Welschschweizern steigert die Spannung in dem Land. Die pazifistischen und sozialistischen Gedanken werden immer populärer vor allem in der Arbeiterschaft. Noch während des Krieges fordert eine Grippeepidemie viele Tote. Bald wird auch in der Schweiz ein Generalstreik ausgerufen.

Während der politischen Kämpfe erleben auch die Frauen große Veränderungen, persönliche Krisen. Gertrud, die einzige Tochter von Amman findet in ihrer Ehe keine Freude, kein Verständnis mehr und sie will sich von ihrem Mann scheiden lassen, auch wenn ihre Mutter deswegen mit ihr den Kontakt nicht mehr pflegt. Sie wird auch verliebt, als sie Pauls Freund, Albin Pfister, einen Dichter, kennenlernt. Aber sie muss wieder ein Ziel im Leben finden, als ihr Geliebter an der Grippe stirbt. Die Frau von Amman, Barbara muss während der ganzen Geschichte ihren Mann unterstützen, auch wenn sie mit seinen Entscheidungen nicht einverstanden ist.

Sofort in dem ersten Kapitel, wo die eigentliche Geschichte anfängt (der kaiserliche Besuch wird vor dem ersten Kapitel beschrieben), geht es um den Verkauf des alten, vornehmlichen Familiensitzes. Die Familie muss danach in eine Mietwohnung umziehen, die sie von dem Händler Stockmeier mieten, aber nach einem Konflikt müssen sie auch die Miete verlassen. Nachdem sie eine Zeitlang in einem Hotel wohnen, kauft Amman ein Grundstück und lässt ein Haus bauen. Der Ausblick von diesem Haus ist schön auf die Stadt, aber Amman will nicht einen großen Haufen Geld für das Grundstück vor dem Haus ausgeben, so wird Stockmeier das kaufen und er lässt ein hohes Gebäude vor dem Haus von Amman bauen. 


\section{Raumkonzepte in der Literaturwissenschaft}

\subsection{Zum Stand der Forschung}

Es ist nicht zu widerlegen, dass der Raum in den letzten Jahrzehnten zu einer wesentlichen Kategorie der geisteswissenschaftlichen Diskurse geworden ist. Wie Wolfgang Hallet und Birgit Neumann feststellen, der Raum ist nicht nur Handlungsort, sondern er verfügt auch über andere Bedeutungen (vgl. Hallet/Neumann 2009, S. 11). In der Literatur finden wir menschlich erlebte Räume, in denen das Kulturelle und die individuellen Erfahrungen mit den räumlichen Umständen zusammenhängen. Der Raum kann in sich selbst Informationen über kulturell vorherrschende Normen, Vorstellung über Marginalität und Zentralität, Werthierarchien usw. vermitteln. Das heißt, die Raumdarstellung steht nicht nur dafür, den Eindruck der Welthaftigkeit zu evozieren, sie erfüllt auch weitere Funktionen. Würzbach geht davon aus, dass Räume als „fiktionaler Baustein, kultureller Sinnträger, Ausdruck der Geschlechterordnung" (Würzbach 2001, S. 105) fungieren. Die Gestaltung des Raumes in einem literarischen Werk kann auch als Komponente sich wandelnder Zeichensysteme verstanden werden (vgl. Nünning 2009), also die Raumgestaltung formt eine Art symbolisches Rahmenwerk, das die Handlung und die Figurenkonstellation übersichtlicher und nachvollziehbarer macht. Aber was die Literaturwissenschaft unter Raum versteht, ist sehr diffus (vgl. Hallet/Neumann 2009, S. 11). Deswegen unterscheiden sich die literaturwissenschaftlichen Raumkonzepte weitgehend. 
Der Raum hat früher wenig Rolle in den Geistes- und Sozialwissenschaften gespielt. Traditionell war die Zeit mit Begriffen wie Mobilität, Progressivität und Entwicklung in Verbindung gebracht, der Raum war dagegen als immobil, fest und stillstehend betrachtet. Damit der Raum als solche der Gegenstand einer Untersuchung werden konnte, mussten zuerst diese Konnotationen überwunden werden (vgl. Schroer 2008, S. 142). Einen Paradigmenwechsel brachte Edward W. Sojas neue Vorstellung über den Raum: das ,spatial turn'. Im Klappentext von Sojas Thirdplace wird festgestellt, dass es in dem 20. Jahrhundert ähnlich großen Wert auf den Raum gelegt wurde, als früher auf die Zeit und auf die sozialen Verhältnisse (vgl. Döring/Thielmann 2008, S. 9). Der Raum wird neu definiert und als eine Widerspiegelung der Machtverhältnisse zwischen den Protagonisten betrachtet (vgl. Hallet/ Neumann 2009, S. 11). Ein anderer wichtiger Denker, der in dieser Neukonzeptualisierung des Raumes Teil hatte, war Michel Foucault. In seinem Vortrag, $A n-$ dere Räume, der später auch publiziert wurde, hat er unter anderem darüber geredet, dass das 19. Jahrhundert alles als zeitliches Nebeneinander betrachtete, während 'unsere Zeit' (was er darunter versteht, wird nicht detailliert) als räumliches Nebeneinander strukturiert wurde (vgl. Foucault 1992, S. 34f).

Der Historiker Karl Schlögel (2003) betont, dass es bei den ,turns' nicht um Neuentdeckungen geht, sondern um Blickwinkelverschiebungen, $d$. h. es wird auf bisher vernachlässigte Aspekte konzentriert. So können ältere Texte wieder als hochaktuell gelten. Wenn man dieses Kriterium betrachtet, trifft es auf die Raumforschung jedenfalls zu. Die Raumforschung ist eine solche Disziplin der wissenschaftlichen Forschungen, die die literarischen, theologischen und kunstgeschichtlichen Werke aus einem neuen Aspekt untersucht (vgl. Baumgartner/Klumbies/Sick 2009. S. 20). 
Grundsätzlich kann es festgestellt werden, dass jedes literarische Werk einen Schauplatz, einen Handlungsort hat, wie das auch Schlögel in seiner theoretischen Arbeit In Räume lesen wir die Zeit (2003) ausführt. Wenn man ein literarisches Werk liest, verbindet man es ganz natürlich mit einem Ort, der sowohl imaginär als auch sehr realistisch sein kann. Es kann also die Frage gestellt werden, warum wählen die Schriftsteller für ihre Geschichte einen nicht-wahrscheinlichen Schauplatz, oder gerade einen präzis lokalisierbaren Ort (vgl. Piatti 2008, S. 16). Die Antwort ist höchstwahrscheinlich das, dass sie auch damit bestimmte Informationen vermitteln wollen. Dass der Raum schon immer eine so wichtige Rolle in der Literatur spielte, mag auch beweisen, dass schon Aristoteles darüber berichtet, in einem Drama sollten die Zeit, der Ort und die Handlung einheitlich sein, und das wurde dann auch in der französischen Klassik maßgebend.

\subsection{Der Begriff des Raumes}

Wichtig ist es, den Begriff des Raumes zu klären, damit man in dem Weiteren daran anknüpfen kann. Im Handbuch von Martínez wird der Raum als ein Objekt der erzählten Welt definiert, das eine „Unterscheidung von innen und außen aufweist und [...] zur Umgebung mindestens einer Figur" (Dennerlein 2011, S. 158) wird oder werden kann. Diese Definition ist zwar richtig, aber nicht genug konkret für meine Untersuchung. Bollnow hat viel über den Raum geschrieben und dabei solche Feststellungen gemacht, die die Rolle des Raumes bzw. des Hauses im Schweizerspiegel sehr genau beschreiben. Im Folgenden referiere ich seine Forschungsergebnisse (vgl. Bollnow 1979). Wenn es über Raum gesprochen wird, denkt man oft an den mathematisch-physikalischen Raumbegriff. Der Raum in 
dieser Sicht ist homogen, jeder beliebige Punkt kann der Mittelpunkt des Bezugssystems werden, so erstreckt sich der Raum von jedem Punkt in die Unendlichkeit. In der Literatur kommt dagegen der erlebte Raum vor. Der erlebte Raum hat einen Mittelpunkt, der von dem Menschen bestimmt wird, der in dem Raum lebt, und so werden auch alle seine Bewegungen auf diesen Mittelpunkt bezogen. Natürlich kann man sich von diesem Punkt entfernen und dann zu ihm zurückkehren. Ob diese Entfernung nur innerhalb des Zimmers ist, oder bis in ein anderes Land geht, ist dabei nicht wesentlich. Die Bewegung des Menschen wird immer von der Dynamik des Fortgehens und Zurückkehrens bestimmt, weil das Bedürfnis nach einem sicheren, vertrauten Platz sehr stark ist und es genetisch einkodiert ist. Das kann auch in solchen einfachen Situationen beobachtet werden, wie eine Konferenz, wo die Menschen nach der Pause unwillkürlich den Platz suchen, wo sie früher gesessen sind, und wenn sie den Platz wechseln müssen, werden sie unruhig.

Die Mitte des erlebten Raumes ist aber selber wieder ein Raum und kein Mittelpunkt im mathematischen Sinn. So wird ein engerer Raum ausgesondert; ein Raum, wo der Mensch wohnt, wo er seine Ruhe findet. Auch die ursprüngliche Bedeutung des Wortes weist auf diese Konzeption hin. In dem Grimmschen Wörterbuch ist zum Verb „räumen” die folgende Bedeutung zu finden, wie „einen raum, d. h. eine lichtung im walde schaffen” (vgl. Grimm 1984, S. 185). Damit man diesen sicheren Aufenthalt hat, braucht man diesen Ort durch Wände abzugrenzen. So gewinnt der Innen- und Außenraum zwei ganz verschiedene Bedeutungen. Der Innenraum ist das Sichere, das Private, der Bereich der Familie. Der Außenraum ist der Ort der Öffentlichkeit, der Ort des Kampfes um das Dasein. Im Leben kann man sich nur im Außenraum entfalten, man 
braucht aber immer wieder die Geborgenheit des Innenraumes (vgl. Bollnow 1979).

Wenn wir in der Bestimmung der Rolle des Hauses in dem menschlichen Leben bei mathematischen Parallelen bleiben, würde ich das Haus mit dem Mittelpunkt eines Kreises vergleichen. Ein Kreis kann nur dann gezeichnet werden, wenn der Mittelpunkt festgestellt ist. Wenn man alle anderen Angaben des Kreises kennt, nur den Mittelpunkt nicht, kann man den Kreis nicht zeichnen. Das Menschenleben braucht auch so einen Mittelpunkt und dieser Mittelpunkt ist der Wohnort. Nur in der Kenntnis des Hauses kann das Menschenleben "gezeichnet" werden; nur dann kann der Mensch sein Leben gut gestalten, wenn er einen sicheren Mittelpunkt in seinem Leben hat.

Diese Definition des literarischen Raumes, bzw. die Rolle des Hauses in dem Raum beschreibt sehr präzis die Rolle der Häuser im Schweizerspiegel. Es ist im Roman tatsächlich zu beobachten, dass das Haus als Kohäsionskraft dient und mit dem Verkauf des alten Hauses, wo diese Kohäsionskraft verschwindet, lockeren sich die Beziehungen und die Konflikte gewinnen Raum. Zusätzlich wird Amman seine Position bei dem Militär verlieren, weil er, nachdem sein eigener Wohnsitz verkauft wurde, die nötige Zuflucht ins Innere zu Hause nicht findet, und wie Bollnow das auch beschreibt, nur wenn ein Gleichgewicht zwischen der im Innen- und im Außenraum verbrachten Zeit entsteht, kann man sowohl im Privatleben als auch in der Karriere erfolgreich sein.

\subsection{Funktionen literarischer Räume}

Der Raum kann in den literarischen Werken verschiedene Funktionen haben je nachdem was der Autor durch die Dar- 
stellung des Raumes vermitteln will. Bei der Analyse des Schweizerspiegel ist es wichtig, die Funktion des Raums zu bestimmen, damit im Weiterem die durch die Raumdarstellung vermittelten Informationen und Deutungen anhand der Funktion des Raums genauer verstanden werden können. Brynhildsvoll (vgl. 1993, S. 8ff) unterscheidet zwischen sechs Funktionen der Raumentwürfe, obwohl diese Funktionen meist nicht als reine Typen auftreten. Er denkt, dass der Raum als Kulisse dienen kann. Der Raum geht aber in den meisten Fällen darüber hinaus und wird zum Resonanzboden für die Emotionen, und fungiert als Projektionsbereich geistig-seelischer Inhalte. Es ist auch möglich, dass der Raum und Mensch völlig auf einander abgestimmt sind, sodass sie sich gegenseitig ergänzen und erklären. Der Raum kann auch eine Schicksalsmacht sein, der die Protagonisten ausgeliefert sind; oder er kann als Bauelement mythischer Weltentwürfe dienen.

Im Schweizerspiegel ist der Raum einerseits die Kulisse des Geschehens, die Emotionen der Protagonisten werden nur wenig durch die Raumdarstellung vorgestellt. Am Anfang, wo das alte Haus vorgestellt wird und über dessen Verkauf geredet wird, werden dunkle Farben verwendet, um das Untergehen einer Epoche und das Trauern der Protagonisten zu betonen. Aber es ist nicht typisch, dass der Raum für die Darstellung der inneren Prozesse steht, sondern eher für die Darstellung bestimmter Charakterzüge. Der Raum kann auch nicht als Schicksalsmacht betrachtet werden, weil die Hauptfiguren zu keinen Eigengesetzlichkeiten von dem Raum gezwungen werden. Der Raum dient also eher als eine Folie, die verstärkt zeigt, was in dem Leben der Familie grundsätzlich passiert.

Roland Innerhofer, der über die Rolle der Architektur in der Literatur forscht und in diesem Thema an der Universität Wien regelmäßig Vorlesungen hält, schreibt in einem seiner Handouts, dass die Architektur im literarischen Text auch als 
Gedächtnisspeicher dienen kann (vgl. Innerhofer 2012, S.3), Gebäude können der Ausgangspunkt zur Erinnerung sein. Genau das gilt auch in dem Schweizerspiegel, wo das alte Haus für die familiären Erinnerungen und die bürgerliche Kultur steht.

\section{Hypothese und die verwendete Methode}

Ausganspunkt meiner Untersuchung ist die Annahme, die auch Hallet und Neumann (2009) formuliert haben, dass der Raum ein „kultureller Bedeutungsträger” (S. 11) ist, wodurch wir "eine konkret anschauliche Manifestation” (ebenda) von Werthierarchen erfahren. Meine Hypothese ist, dass im Schweizerspiegel durch die Beschreibung der Häuser die Art und die Änderung der Beziehungen innerhalb der hausbesitzenden Familie dargestellt bzw. angedeutet werden. In meiner Untersuchung konzentriere ich mich auf die drei Wohnsitze der Familie Amman, in einem Kapitel mache ich aber auch einen Ausblick auf andere Häuser im Text.

Wie Matt in dem Nachwort zum Schweizerspiegel auch festgestellt hat, finden wir im Roman keine detaillierten Stadtbeschreibungen, die Schauplätze haben Modellcharakter und geographisch werden sie kaum benannt. Dagegen wird das alte Haus der Famile Amman sehr detailliert beschrieben und auch über das neue Haus erfährt man wichtige Informationen.

In meiner Untersuchung greife ich auf die Raumdefinition von Bollnow zurück (1979, S.15), der den mathematischphysikalischen Raum von dem erlebten Raum unterscheidet. Bollnow geht davon aus, dass in den literarischen Werken der Raum einen Mittelpunkt hat, auf den sich alle Bewegungen der Protagonisten beziehen. Er stellt fest, dass dieser Mittelpunkt 
oft das Haus ist, das die Identität, den gesellschaftlichen Status ausdrückt, das die Innen- und Außenwelt trennt.

Bei der Untersuchung des Romans stütze ich mich in erster Linie auf den Text selbst, aber bei der textnahen Interpretation konzentriere ich mich auch auf die Aspekte, die in der Sekundärliteratur beschrieben werden. In meiner Erkl, arung befasse ich mich hauptsächlich mit den Stellen, wo es um den Wohnort der Familie geht.

\section{Interpretation}

\subsection{Das alte Haus}

Die Wichtigkeit des Hauses als Symbol im Schweizerspiegel wird schon dadurch betont, dass es in dem ersten Kapitel nur um den Verkauf des Wohnsitzes der Familie Amman geht. ${ }^{1}$ Weiterhin werden die Beuhausungen der Protagonisten in jedem Fall vorgestellt, obwohl Inglin in seinem Werk nur begrenzt über die Umgebung schreibt. Wenn über das alte Haus gesprochen wird, wird oft nicht nur das Haus selbst thematisiert, sondern auch die alte bürgerliche Tradition. Das ist gut bemerkbar in dem Gespräch zwischen Amman und dem Anwalt der Genossenschaft, die Ammans Haus kaufen will. Der Anwalt sagt: „ein altes Haus an einem solchen Platze [ist] nicht zu retten" (Inglin 2014, S. 17) ${ }^{2}$ und er weist auf das Haus mit den Worten „feudaler Sitz” (ebenda) hin. Später wird das Haus

1 Das Buch fängt mit einem „Vorspiel” an, wie Matt (2014) das in ihrem Nachwort bezeichnet (S. 884). Dieses Vorspiel ist eine Szene, die nicht konkret weitergeführt wird, aber über eine wichtige Bedeutung verfügt. Diese Bedeutung zu analysieren, könnte das Thema einer anderen Arbeit sein.

2 Im Folgenden werde ich bei den Zitaten nur die Seitenzahlen des Romans angeben. 
den „letzten sichtbaren Zeugen einer vornehmen bürgerlichen Kultur" (S. 19) genannt und beim Abschiedmahl, wo die Familienmitglieder noch zu einem letzten $\mathrm{Mal}$ in das alte Haus eingeladen werden, sagt Hartmann, der Mann von Gertrud, in seinem Toast das Folgende:

Das Haus Amman ist mir immer als eine Verkörperung des guten schweizerischen Bürgertums erschienen, zu dem wir schließlich alle gehören. Seine Tugenden haben sich in diesem Hause bewährt, und bewähren sich immer noch. (S. 90) 3

Das Haus als alter bürgerlicher Wohnsitz steht für das alte System, das von den Jugendlichen immer mehr kritisiert wird. Die Gegenüberstellung des Alten und des Neuen wird schon im ersten Kapitel mit verschiedenen Mitteln hervorgehoben. Als Amman nach Hause geht, biegt er in eine „breite, geräuschvolle Straße ein” (S. 18) und bald "gewährt [er] [...] einen bescheidenen Blick ins Innere des stillen Gutes" (S. 19). Die moderne Welt ist laut, das alte Haus ist still. Ein weiterer Unterschied ist in dem Aussehen der Häuser zu finden. Ammans Haus hat ein kunstvolles, schmiedeeisernes Gittertor, alte Parkbäume „auf einer Länge von achtzig Schritten” (S. 19), eine Säulenvorhalle und die ,edle[n] Verhältnisse [ließen] [sich] im Licht des späten Nachmittags [...] erkennen” (S. 19), dagegen werden die Miets- und Geschäftshäuser einfach als "geschmacklos” (S. 19) gekennzeichnet. Später wird Ammans Haus noch detaillierter beschrieben: Dort ist alles sehr nobel, elegant, sogar aristokratisch, was alles früher noch eine wichtige Rolle im Leben des Bürgertums spielte, jedoch ist diese Schönheit in dem neuen

3 Der Ausdruck „Haus” steht nicht nur für das Gebäude selbst, sondern wie in diesem Fall metonymisch auch für die Familie Amman. 
Jahrhundert nicht mehr so wichtig, die Handlichkeit ist viel wichtiger.

Als Amman von der Besprechung über den Hausverkauf heimkehrt, tritt er durch die schmale Seitenpforte in den Garten. Das Tor mit dem Wappen wurde längst nicht mehr geöffnet, was darauf hinweist, dass das bürgerliche Leben seine Bedeutung verloren hat, die ehemalige Pracht wurde durch die Zweckmäßigkeit ersetzt. Das Wappen auf dem Tor ist nicht Ammans Wappen, was auf eine Entfremdung zwischen dem Hausherrn und dem Haus hinweist. Diese Entfremdung kann eine Folge des Zeitgeistes sein, aber auch ein Verursacher des Verkaufes. Als Amman in den Garten hineingeht, sieht er mit einem „betont gleichgültigen Blick” (S. 19) auf das Wappen und stießt die Seitenpforte hinter sich hart ins Schloß. Diese Reaktionen spiegeln seine innere Entfremdung vom Haus. Ob sie eine unwillkürliche oder bewusst gerichtete Entfremdung und Distanzierung ist, wird nicht eindeutig markiert.

Mit dem Abbruch des Hauses wird also nicht einfach nur ein altes Haus vernichtet, sondern auch, wie die Bezeichnungen und Andeutungen das auch zeigen, das alte System: die Tradition der konservativen Bürger. Auch Schneider-Handschin hebt diese Bedeutung des Hausverkaufs hervor, als sie in ihrer Studie feststellt, dass der „Hausverkauf [...] auf den Zerfall der 'bürgerlichen Kultur' als Folge der Zeit [verweist]" (SchneiderHandschin 1997, S. 148). Am klarsten wird die tiefe Kluft zwischen den Arbeitern und der Familie, die zum Bürgertum gehört, dort gezeigt, wo Paul und Barbara im Garten spazieren und sie die eiligen Arbeiter beobachten, die nach der Mittagspause in die Arbeit zurückgehen. Paul und Barbara sehen „plötzlich durch die Gitterstäbe” das „verächtlich spähendes, dunkles Gesicht” eines breitschultigeren Burschen und „hörten auch die häßliche Bemerkung, mit der er sich wieder den übrigen anschloß”. (S. 43) Inglin führt diese Szene nicht weiter, der 
letze Satz des Kapitels ist einfach nur der Folgende: „Schweigend kehrten sie um". (S. 43) Aber diese kleine Szene zeigt sehr gut, dass die Familie Amman zu den Bürgern gehört. Ein Gitter steht zwischen den Arbeitern und den Bürgern und das Haus mit dem Garten und Gitter schützt das Bürgertum. Als das Haus verkauft wird, wird dieser Schutz aufgegeben, die Bürgerfamilie wird Problemen ausgesetzt und gerät in Konflikten mit anderen Sichtweisen der Gesellschaft. Das zeigt wie die alte bürgerliche Kultur langsam zerfällt. Ein weiterer Aspekt ist, dass Paul sich die ganze Zeit im Roman bemüht, sich an den Arbeiterbewegungen beteiligen zu können, aber diese kuze Szene spiegelt sein Schicksal wider, dass er nie von den Arbeitern völlig akzeptiert wird. Als Bürger kann er nie die Kämpfe der Arbeiter verstehen, weil er selber nie darauf angewiesen ist zu kämpfen, seine Existenz wird nie gefährdet, seine Zukunft wird trotz all den Problemen nie fragwürdig werden. Wie das Gitter ihn von den Arbeitern in dieser Szene trennt, so trennt ihn sein bürgerliches Sein von den Arbeitern im Leben.

Aber auch die Identität der Familie wird durch den Abbruch des Hauses in Gefahr geraten. Baumgärtner, Klumbies und Sick stellen in der Einführung ihres Sammelbands über die Raumkonzepte fest, dass „Kulturräume [...] Ansatzpunkte für Identitätsstiftung und Zuordnungen [liefern]" (Baumgärtner/Klumbies/Sick 2009, S. 10). Ähnlicherweise trägt das vornehme bürgerliche Haus dazu bei, dass die Familie ihre Identität formen und bewahren kann, aber genau diese Identität wird durch den Hausverkauf in Frage gestellt. Das ist auch der Familie schon von Anfang an bewusst. Vor allem ist Barbara diejenige, die das weiß und darunter leidet. Sie war als Frau und Mutter immer dafür zuständig, ein Zuhause für ihre Familie zu schaffen. Sie denkt, das Haus war das, das „die zerstreute Familie [...] immer wieder umschloß”, es war ein "geheimnisvolle[s] alles umfassende[s] 'Daheim' “, und obwohl sie nichts „gegen 
die Entwicklung der Stadt” einzuwenden hatte, fragt sie sich „warum [...] man sich dieser Entwicklung nicht entziehen [konnte], wenn man genug davon hatte?” (S. 24). Die Entwicklung der Welt hat also das Alte „rücksichtslos hinweggestampft" (S.24). Und beim Abschiedsmal kommt die Identitätskrise der Familie am klarsten zum Ausdruck, wo das

Gerede von zerfallender Form, von Zukunft [...] ihren [Barbaras] Blick [...] zurück [lenkte], und statt von heiterer Zuversicht [...] sie von der trüben Ahnung erfüllt [war], daß hier eher etwas ende, eine Ammansche Epoche sozusagen, eine glänzende Epoche, deren Fortsetzung auf jeden Fall problematisch geworden war. (S. 90)

Ähnlicherweise fühlt Amman einen „dunkle[n] Widerstand”, weil er weiß, dass „er mit dem Familiensitz mehr verkaufen werde, als einen guten Bauplatz” (S. 18). Sein Sohn, Paul vertritt auch diese Meinung, weil er sagt: „Er [Amman] hat nie gewusst, was er hier besaß, und daß es so etwas nicht zum zweitenmal gibt" (S. 42)

Das alte Haus hatte eine wichtige Rolle in dem Zusammenhalt der Familie. Das Haus war groß genug extra ein Musiksalon zu haben, wo Quartett gespielt werden konnte. Im Quartett spielen normalerweise Severin und Paul, die zwei Söhne von Amman, die oft Konflikte miteinander haben; Gaston Junod, der französisch-schweizerische Verwandte und Gertrud. Die Konflikte werden natürlich nicht vergessen, aber das Haus bietet einen Ort, wo man trotz der Meinungsunterschiede gemeinsam etwas zustande bringen kann. Das sieht man gut, als sie einmal zum Spielen zusammenkommen und Severin mit seinem Vater verurteilend über Pauls Haltung redet. Er sagt, er würde an seines Vaters Stelle sich das nicht gefallen lassen, was Paul macht (S. 57), und gleich danach ist er Freds Meinung 
nach „ekelhaft” (S. 60) beim Spielen und kritisiert Paul oft, trotzdem spielen sie weiter. Später, als Barbara ein letztes gemeinsames Familienessen im alten Haus organisieren will, ist das Musikspielen der Anlass des gemeinsamen Essens. Dieses Mal spielen sie mit dem Bewusstsein, dass sie in diesem Raum zum letzten Mal spielen können, in dem Raum, „der ihr bei aller Unvollkommenheit der Musikanten doch eine bescheidene Heimat gewesen war" (S. 96). Genau diese Heimat werden sie mit dem Haus verlieren und dadurch alle Vorteile, die eine Heimat bieten kann: die Sicherheit, die Ruhe, den Frieden und die Geborgenheit.

Die Salonmusik oder Hausmusik spielte in dem 19. Jahrhundert eine wichtige Rolle in der bürgerlichen Kultur. Die Salons waren eine Art Mittelstellung zwischen dem Privaten und Öffentlichen (vgl. Ritter 2004, S. 41). Familienmitglieder, Bekannte, Freunde konnten eingeladen werden; das private Musizieren der Familie wurde einem größeren Publikum zugänglich gemacht. Das alte Haus, das ein bürgerliches Haus ist, wurde so gebaut, dass dieses Salonleben dort möglich war. Die Familie hat diese Möglichkeit genutzt um gemeinsam zu musizieren, so haben sie noch eine bürgerliche Tätigkeit ausgeübt. Das wird aber nach dem Hausverkauf nicht mehr möglich, weil die Mietwohnung zu klein ist und in dem neuen Haus kein Musikzimmer zu finden ist. Die „Musiker” der Familie versuchen noch nach dem Hausverkauf andere Alternative zu finden, aber das funktioniert wegen den Familienkonflikten nicht. Das betont wieder, dass das alte Haus für dem familiären Frieden und für die bürgerliche Kultur stand.

Für Gertrud hat das alte Familienhaus eine besondere Rolle. Als sie zum Nachtessen zum letzten Mal ins Elternhaus eingeladen wird, wohin sie auch ihren Mann mitnehmen soll, überlegt sie, was ihr das Haus bedeutet. Sie kennt im Garten „jedes Winkelchen” (S. 76f) und sie spürt, dass „sie noch immer mit 
ganzer Seele” (S. 77) an dem Haus hängt. Es ist „ein Stück ihres 'Reiches', ihres ganz persönlichen inneren Reiches, zu dem ihr Mann keinen Zutritt” (S. 77) findet. Mit dem Abbruch des Hauses verliert Gertrud nicht nur das Familienhaus, ihre seelische Heimat, sondern auch ihre Hoffnung auf ein besseres Leben.

Dass mit dem Abbruch des Hauses etwas Wichtiges zugrunde geht, wird auch mit der Hilfe der Farben betont. Im ersten Kapitel stehen Ausdrücke, wie „schwarze[...] Ranken und Stäbe”, „späte[r] Nachmittag” (S. 19) und später als Frau Barbara Ammans Entscheidung über den Verkauf des Hauses erfährt, sitzt sie „im schon fast dunklen Zimmer [...] und [denkt] nicht daran [...] Licht zu machen” (S. 24), sie blickt „in den Garten hinab, der in einem seltsamen Zwielicht” (S. 24) liegt und die Gebüsche schaffen „ein dichtes Dunkel” (S. 24) im Hintergrund. Aber auch die Geräusche werden dafür verwendet, die Tragödie des Hausabbruchs zu betonen. Beim Abschiedsmal freut sich Albin, Pauls Freund, wie die Musik im Musiksalon klingt. Darauf antwortet Paul sofort mit bitterem Geschmack im Munde. „Ja... in vierzehn Tagen werden hier andere Töne erklingen [...] Es wird prasseln, splittern, krachen..." (S. 93) sagt Paul. In den nächsten Sätzen detailliert er noch diese Töne und redet er auch über die Gerüche.

Wenn das Haus für alle so besonders und wichtig war, kann natürlich die Frage gestellt werden, warum sie das Haus doch verkaufen wollten. Die Antwort liegt wahrscheinlich genau darin, dass sie sich selber für modern halten und nicht in der Vergangenheit stecken bleiben wollen. Der Anwalt weist darauf hin, dass die Stadt sich entwickelt und es wäre gegen die Vernunft, diese Entwicklung mit einem alten Haus zu behindern (S. 17). Später wird es auch öfters erwähnt, dass Amman und Barbara, beide in ihren Kreisen als modern gelten. „Er hatte auf Grund fortschrittlicher Anschauungen [...] seine vier Kinder 
nicht allzu streng erzogen” (S. 20) - heißt es über Amman. Aber auch Barbara ist für Neuerungen aufgeschlossen: „Sie hatte gegen die Entwicklung der Stadt nichts einzuwenden, so wenig wie gegen den Fortschritt überhaupt [...], sie galt in ihren Kreisen denn auch als fortschrittliche Frau, [...] sie hatte an der Seite ihres Mannes gekämpft und gelitten.” (S. 24). Das Ehepaar ist also modern und sie versuchen mit dem Wandel der Welt schrittzuhalten. Deswegen ist es für sie nicht so unvorstellbar, das Haus zu verkaufen. Durch diese Tat wollen sie der Welt, aber auch sich selbst zeigen, dass sie nicht gegen die Änderungen sind.

In ihrem Verhalten steckt jedoch eine gewisse Ambivalenz. Einerseits verkaufen sie das Haus, andererseits war der „dunkle Widerstand” (S.21) „der einzige konservative Rückstand in” (S. 19) Ammans Wesen. Ähnlicherweise sagt Paul, dass Amman nie gewusst hat, was er besaß (S. 42), aber kurz danach sagt er, dass sowieso alles dahingeht (S. 42). Das zeigt, dass das Haus für Paul doch einen besonderen Wert hat, obwohl er linksorientiert ist. Bezeichnenderweise versucht die ganze Familie zu leugnen oder verstecken, dass sie teilweise konservativ sind.

Ein anderer Grund, warum das Haus verkauft wird, ist das Ehren des Geldes. Die bürgerliche Familie und das Bürgertum überhaupt hat sich sehr an die Bequemlichkeit des Wohlstandes gewöhnt. Das wird sowohl explizit als auch implizit markiert. Einerseits ist es oft zu lesen, dass die Protagonisten wohlgenährt sind. Das weist auf den Wohlstand hin, der ermöglicht, immer zum vollen Tisch zu sitzen. Aber auch eine Fünfzimmerwohnung in der Stadt zu mieten ist ein Luxus. Andererseits wird über Barbara geschrieben, dass sie das Geld „sehr zu schätzen wußte”, denn „sie hatte ihr Leben lang im Wohlstand gelebt und gewisse verächtliche Redensarten über den Wert des Geldes immer mit einem Achselzucken abgetan" (S. 24). Der Wohlstand und das Geld spielte also eine wichtige Rolle in der 
Familie, genau deswegen führt Amman den Verkauf des Hauses folgendermaße auf: „Jaa ... [...] das ist mehr als ich erwartet hatte, offen gestanden. Jetzt heißt es zugreifen." (S. 22) Genau diese Einstellung bestimmt ihre Entscheidungen auch in der Zukunft. Als Amman später ein neues Grundstück kauft und überlegt, ob er das andere Grundstück wegen der schönen Aussicht kaufen sollte, denkt er am Ende, dass es da um eine Geldfrage geht, und in "Geldfragen hatte er sein Leben lang sachlich gedacht und gehandelt” (S. 580), dadurch ist er ein wohlhabender Mann geworden. Hier erinnert er sich an den Hausverkauf zurück und er hält das noch immer „für die vernünftigere Lösung, obwohl er die Folgen nicht eben rühmen" (S. 580) kann. Das zeigt sehr gut, dass die Gefühle bei seinen Entscheidungen keine Rolle spielen und er immer vernünftig handelt. So war es möglich, dass er sein Haus und quasi dadurch den familiären Frieden verkauft hat.

Ein weiterer Grund für den Verkauf des Hauses ist eher schriftstellerisch motiviert. Wie es schon früher erwähnt wurde, symbolisiert das Haus das Bürgertum, das diese Zeit in Krise geraten ist. Das wird oft explizit beschrieben und hervorgehoben. Besonders Pauls Figur ist dafür geeignet, die Schwachpunkte des Bürgerstums darzustellen, weil er selber politisch links orientiert ist. Paul macht solche Bemerkungen, wie „Wir leben im Paradies, mein Lieber! Aber dieses Paradies stinkt zum Himmel” (S. 149), „Papa aber lebt durch seine bürgerliche Umgebung, die ebenso fragwürdig ist wie er. Wirf ihn aus Amt und Würden, was bleibt dann? Ein Bürger? Aber was ist das?” (S. 193) und denkt das Folgende „Es ist eure [Ammans] Welt, die zu brennen anfängt und hoffentlich einstürzen wird, eure zivilisierte, sichere, fortschrittliche Welt!" (S. 164). Auch sein Bruder, Fred denkt, ,es wird sich auch in der Zukunft nichts ereignen, das wert wäre, erlebt zu werden" (S. 204). Genau deswegen feiert die Jugend den Ausbruch des 
Krieges. Ihre allgemeine Einstellung ist, dass „jetzt [...] eine neue Zeit mit neuen Maßstäben, neuen Verhältnissen und unverhofften Möglichkeiten [beginnt]" (S. 234). Die Jugend betrachtet diese Änderungen als nötig und erwünscht, dagegen lähmt Ammans Fassungsvermögen „dies furchtbar Zögernde einer fast handgreiflich gewordenen unvorstellbaren Katastrophe” (S. 222) und „erfüllte ihn mit tiefer Unsicherheit” (S. 222). Die Änderungen der Zeit sind unaufhaltsam, das wird im Roman eindeutig vorgestellt und deswegen ist es unvorstellbar, dass ein bürgerliches Haus, das für das alte Bürgertum steht, weiterhin bewahrt wird. Das wäre mit der Geschichte unvereinbar, also der Hausverkauf wurde nicht nur innerlich motiviert, sondern auch gleichsam durch die Logik der Ereignisse erzwungen.

Aber das alte Haus steht im Roman nicht nur für die Identität der Familie, bzw. für das traditionelle Bürgertum, sondern auch für das ganze Land. Darauf macht uns Amman aufmerksam, als er beim Abschiedsmahl eine Rede als Antwort auf die Rede seines Schwiegersohnes hält. In dieser Rede weist er auf das Vaterland hin und sagt: „wir wollen nicht vergessen, wem wir alle unser Wohlergehen und unsere Sicherheit zu verdanken haben.” (S. 91) Und die „Entwicklung geht weiter, [...] ein Rückfall ist nicht mehr denkbar, der Fortschritt ist unaufhaltsam" (S. 91). Amman weiß, dass sein Leben mit der Schweiz und mit dem Schicksal des Landes eng verbunden ist, aber er glaubt an den Fortschritt und daran, dass er mit dem Verkauf des Hauses in eine fortschrittliche Zukunft geht. Ihm ist es bewusst, dass er mit dem Haus „die Vergangenheit” verkauft und er hofft, dass es zu etwas Besserem führen wird. Was er aber nicht ahnt, dass in der Zukunft die Familie ihr richtiges Zuhause und inneren Frieden verlieren wird und ähnlicherweise auch das Land in eine schwierige Situation gerät. 


\subsection{Miete bei Stockmeier}

Die Miete bei Stockmeier in der Dufourstaße scheint für die Familie die beste Lösung zum Wohnen nach dem Verkauf des alten Wohnsitzes zu sein. Damals gab es kein geeignetes vergleichbares Haus in der Stadt und Amman fand, dass ein neues Haus zu bauen übereilt wäre. (S. 22) Das scheinbar einzige Problem mit der Wohnung ist, dass da "ein Zimmer zu wenig” (S. 23) ist. Schon das führt zu einem Konflikt, der aber ein Vorspiel der zukünftigen Ereignisse und Verhältnisse ist. Amman denkt, Paul braucht in der Wohnung kein Zimmer zu haben, weil er eh am Graberschen Institut sein wird. (S. 23) Aber Barbara macht sich Sorgen, weil er noch nicht da ist und sie auch kein Gästezimmer haben. In der Zukunft werden allerdings Konflikte in der Familie entstehen und dadurch werden Paul, aber auch Gertrud unerwünschte Gäste bei den Eltern; und auch die welsch-schweizer Familienmitglieder werden sich nicht mehr mit den Ammans treffen wollen. Dass sie so eine Wohnung mieten wollen, wo es "ein Zimmer zu wenig” (S. 23) ist, zeigt, dass sie in der Zukunft tatächlich weniger Platz brauchen werden. Aber Barbara, die sich als Mutter dafür verantwortlich fühlt, ein richtiges Zuhasue zu schaffen, verhandelt von Stockmeier ein zusätzliches Zimmer. (S. 43) Das zeigt, dass sie ihr Bestes für den Frieden tut, und wenn es nötig ist, auch zwischen den Familienmitgleidern vermittelt, das in der $\mathrm{Zu}-$ kunft von großer Bedeutung sein wird. Mag die Wohnung in der Dufourstraße aber eine gute Lösung sein, blieb sie doch für die Familie immer nur eine Miete, wo die Familie sich nie wirklich wohlfühlen kann. Schon bevor die Wohnung gemietet wird, ist ein ungleiches Verhältnis zwischen Stockmeier, dem Vermieter, und Amman, dem Mieter zu spüren. Amman „bewahrte aber jene Zurückhaltung, die er im Verkehr mit einfachen Leuten seinem öffentlichen Ansehen und seiner Stellung 
schuldig war" (S. 34) und seine Beziehung mit Stockmeier wird mit der Zeit nicht besser.

Stockmeier, ein wahrer Händler, versucht jede Situation zum eigenen Vorteil zu nutzen. Als der Krieg ausbricht und die Leute Waren aufhäufen wollen, nützt Stockmeier die verzweifelten Menschen aus und verkauft alles zu höherem Preis. Und genau diese Einstellung ist in seiner Beziehung zu Amman zu beobachten. Er hofft, politische Nachrichten aus Amman herauszukriegen, als Amman aus Bern heimkehrt (S. 182f) und hofft, dass Amman für seinen Sohn, Leo, der sich in der Offizierschule nicht würdig verhalten hat, vermittelt. (S. 501) Seine Frechheit kulminiert, als er die das Abendessen verzehrende Familie in Hauspantoffeln besucht. Als wenn er kein Gast wäre, sondern zur Familie gehören würde. Diese bewusste, unverschämte Außerachtlassung der Intimsphäre der Famlie und die bewusste Betonung seiner Macht im Haus, ist eine Untermauerung des Gefühls, dass sie zwar in der Wohnung wohnen, aber nicht wirklich zu Hause sind. Genau diese unangenehmen Begegnungen machen es unmöglich, sich in der Wohnung wirklich wohl zu fühlen, und diese Spannung und Unruhe um die Wohnung ist wie ein Spiegelbild der Verhältnisse in der Familie.

Schon das erste Bild, wo die Familie endgültig in das neue Haus eingezogen ist, ist mit Übelkeit verbunden. Auch wenn es nicht wegen dem Haus ist, assoziiert man vom ersten Augenblick an etwas Negatives mit der Mietwohnung. „Fred erwachte in der neuen Wohnung an der Dufourstraße zur gewohnten Zeit mit schwerem Kopf und einem faden Geschmack im Munde" (S. 97) So lautet der erste Satz des zweiten Teiles des Romans und dieser Satz ist auch der erste, der über das Leben in der neuen Wohnung berichtet. Der Grund für Freds Übelkeit ist, dass er am Abend vorher zu viel getrunken hat, als er den Schluß des Wintersemesters gefeiert hat. Trotzdem steht er früh auf, weil er sofort zu seinem Onkel im Rusgrund fahren 
will. Also kaum ist er zu Hause, will er sofort weg. Das zeigt, dass er sich in seinem neuen Zuhause nicht richtig wohl fühlt und im Weiteren erfahren wir, dass er tatsächlich viel lieber auf dem Lande bei seinem Onkel ist.

Später wird eine familiäre Szene in der Wohnung (eine der wenigen) dargestellt und diese Szene ist auch mit Spannung belastet. Schon am Anfang lesen wir, dass Barbara das Dienstmädchen in die Küche schickt und sie den Tisch selber mit finsterer Miene deckt. Aus Ammans Büro dringt ein "heftiger Wortwechsel” (S. 436), weil Amman mit seinem welschschweizer Schwager über den Krieg diskutiert. Nach dem Abtritt des Verwandten herrscht beim Tisch weiterhin eine unangenehme Spannung. Fred, Paul und Amman diskutieren vehement über den Krieg und sie beenden das Mittagessen früher, als sonst. Am Ende bleibt nur Barbara zurück und sie zählt die zahlreichen Probleme in der Familie traurig auf. Sie kann aber die „hintergründige Strömung dieser Zeit, d[ie] elementare Auflockerung des Lebens” (S. 441), die sie "an der eigenen Familie erfuhr" (S. 441) weder annehmen, noch begreifen. Die ganze sichere Welt der Familie droht auseinanderzufallen. Die Familie hat vor kurzem nicht nur das eigene Haus verloren, sondern auch den Frieden, den die Familie und das familiäre Haus bot.

Mit der Zeit wird die Beziehung der Familienmitglieder immer weniger friedlich. Severin der älteste Sohn hat die bürgerlichen Erwartungen schnell erfüllt, er heiratete eine bürgerliche Frau, erzeugte Kinder und hat eine Arbeit. Er redet gern über die Politik, dadurch hat er die beste Beziehung zu Amman unter den Kindern. Aber die deutschen Ereignisse begeistern ihn immer mehr, seine politischen Anschauungen werden radikaler und dadurch verschlechtert sich seine Beziehung zuerst mit den französisch-schweizerischen Verwandten, später auch mit seinem Vater. Als der Krieg ausbricht, muss Amman 
in den Nationalrat nach Bern fahren. Severin besucht ihn sofort, als er von Bern heimkehrt und möchte mehr über die politische Lage wissen. Auch einen Artikel zeigt er seinem Vater und will Ammans Meinung darüber hören. In diesem Gespräch haben der Vater und Sohn zum ersten Mal größeren Meinungsunterschied. Amman meint, das Volk sollte in erster Linie beruhigt werden, aber Severin bestreitet dies und sagt, dass das Volk Recht hat alles zu wissen, und er meint, in Bern werden bestimmte Informationen geheim gehalten. (S. 186f) Mit der Zeit wird Severin immer extremistischer und wegen seiner politischen Stellungnahme, die er als Redaktionsdirektor vor der großen Öffentlichkeit bekannt macht, gerät Amman in eine unangenehme Situation im Nationalrat. Amman wird auch dazu gezwungen, über die Zukunft der Zeitung Ostschweizer und dadurch über die Zukunft seines eigenen Sohnes $\mathrm{zu}$ entscheiden. Obwohl er zutiefst unzufrieden mit Severins Haltung ist, kann er seine menschlichen und väterlichen Gefühle nicht außer Acht lassen und möchte irgendwie die $\mathrm{Zu}$ kunft seines Sohnes versichern. (S. 556f) Aber ihr Verhältnis wird nie mehr so harmonisch, wie früher.

Die Beziehung zwischen Amman und Paul war nie perfekt. Paul hat seine eigenen Ideen und er verurteilt das Bürgertum, auch wenn er daraus stammt. Genau das kann sein Vater nicht begreifen. Als Amman mit Barbara in die Mietwohnung zieht, ist der Vater-Sohn-Konflikt schon so ernst, dass Amman sich entschließt, ,auf Paul keine Rücksicht mehr zu nehmen” (S. 33). Der Grund dafür ist, dass Paul nicht rechtzeitig heimgekommen ist, um an dem Wiederholungskurs teilzunehmen, und er sich nicht für die Arbeit angemeldet hat, die sein Vater für ihn versicherte. Sein Einwand dagegen ist: „Ich kann doch nicht, als Einpauker beginnen” (S. 41). Später kann er als Redakteur beim Ostschweizer arbeiten, auch weil Amman es ihm ermöglicht, aber er macht seine Arbeit nicht gewissenhaft und 
mag diese Arbeit überhaupt nicht. Letztendlich als Severin einen Artikel von ihm nicht publiziert, kündigt er und lässt seinen Artikel anderswo erscheinen. Dadurch fühlt sich seine Familie beleidigt und beschämt. Diese Entfremdung geht so weit, dass Paul nicht mehr nach Hause geht, er mietet eine eigene Wohnung und als er an der „spanischen Grippe” erkrankt, will er nicht lassen, dass Barbara ihn zu sich heimnimmt, weil er Angst hat, dass er dafür seine politische Überzeugung verleugnen muss. Als Gertrud ihn darauf aufmerksam macht, dass das alles für die Eltern vielleicht schmerzhaft ist, antwortet Paul mit den einfachen Worten, dass er nicht mehr den gehorsamen Sohn spielen will (S. 457) und als er seinen Vater bei einem Begräbnis sieht, blickt er seinem Vater mit „kühler Neugier nach” und fühlt sich „weit weg von ihm in einer andern Welt" (S. 662). Seine Entfremdung von der Familie scheint endgültig zu sein, er hat ja schon von Anfang an gegen die bürgerliche Welt rebelliert, die seine Familie vertritt. Pauls Trennung von der Familie hängt natürlich nicht nur von ihm ab. Einmal sagt Barbara ihrem kleinsten Sohn, Fred, dass Paul es mit seinem Vater verspielt hat. Auch Amman verschließt sich vor seinem Sohn. Barbara meint, Paul hat eine große Geschichte aus etwas gemacht, ohne Rücksicht auf seinen Vater, und das kann ihm Amman nicht verzeihen. Das zeigt, dass die Konflikte die innersten Bereiche der Seele der Familienmitglieder berühren, und das macht sie blind. Durch den Verlust des Heimes fühlen sich die Protagonisten überall in Gefahr, sie versuchen nicht zusammenzuarbeiten, sondern wollen ihre eigenen Welten schützen.

Auch Gertrud hat Konflikt mit den Eltern. Als sie sich für die Scheidung entscheidet, will Barbara nicht mehr mit ihr reden. Wegen der politischen Stellungnahme entsteht ein tiefer Graben zwischen dem französisch-schweizerischen und der deutsch-schweizerischen Seite der Familie. Gaston will nicht 
einmal zum Musizieren mit den Söhnen Ammans zusammenkommen.

Die Familie Amman hat jahrzehntelang als eine ganz normale, zufriedene, bürgerliche Familie gelebt, aber als sie das alte Haus verkaufen, das Haus, das nach der Raumdefinition von Bollnow immer als ein Bezugspunkt für die Bewegungen der ganzen Familie diente, das der Innenraum für sie war, auch für die Kinder, die die tiefste Ruhe hier finden konnten, verliert die Familie den Halt und bewegt sich Richtung Zerfall. Wie Bollnow feststellt, braucht man einen sicheren Innenraum, wo man Ruhe und Verstärkung findet, damit man im Außenraum eine gute Leistung haben kann (vgl. Bollnow 1979). Wenn Amman sich für den Verkauf des Hauses entscheidet, verkauft er sozusagen diesen Innenraum, und obwohl er versucht für einen anderen zu sorgen, wird die Mietwohnung nie diese Rolle erfüllen können. Deswegen ist es nicht überraschend, dass die Familie, die keine Geborgenheit mehr hat, sich gegen die Konflikte des Außenraumes nicht mehr schützen kann. So passiert, dass Amman während einer militärischen Übung einen Fehler begeht und dadurch seine Arbeit bei dem Militär verliert.

Interessant ist, dass diese Zeit nicht nur die Familie Amman ihr richtiges Zuhause aufgibt. Gertrud, die nicht mehr mit ihrem Mann leben will, zieht in eine eigene Miete mit ihren zwei Kindern, aber auch Paul mietet eine eigene Wohnung, weil er nicht mehr bei seinen Eltern wohnen will. Wie die Familienmitglieder ihre bisherigen Behausungen hinter sich lassen, zeigt, wie sie sich von dem ehemaligen Leben abwenden. Langsam zerstückelt sich die Familie auf Parteien, die gegeneinander und nicht miteinander im Trubel der Zeit kämpfen. Ohne einen inneren Raum, den das alte Haus bieten konnte, scheinen diese Konflikte unauflösbar zu sein.

Als die Konflikte in der Familie auftauchen, bricht auch der Erste Weltkrieg aus. Ob die Konflikte wegen dem Krieg entste- 
hen, oder der Krieg nur die latent bereits vorhandenen zum Vorschein bringt, ist nicht eindeutig. Ihr Zusammenhang mit dem Krieg ist aber unbestritten. Viele Familien in Europa haben ihr Haus wegen des Krieges verloren, also der Krieg war für den Verlust des Innenraums und damit für den Verlust der Familienruhe verantwortlich. In der Schweiz war es aber anders. Das zeigt auch der Roman durch die Ereignisse im Leben der Familie Amman. Wie es schon festgestellt wurde, wurde das Haus wegen dem Geld und wegen dem Glauben an den Fortschritt verkauft. Daraus schließe ich darauf, dass der Autor mit dem Verkauf des alten Wohnsitzes einerseits die Schwierigkeiten im Familienleben andeuten wollte, also dass Meinungsunterschiede und Konflikte das Leben der Familie erschweren werden, andererseits wollte er die Basis dafür sichern, dass die Familie einen neuen Bezugspunkt und dadurch eine neue Art von Lebenseinstellung haben kann. Der Verkauf hätte ohne den Krieg auch stattgefunden und einige Probleme wären sowieso vorgekommen, aber die Familie mit ihren Problemen ist eine gute Widerspiegelung der ganzen politischen und gesellschaftlichen Lage Europas während des Kriegs und diese "Heimatlosigkeit”, “Orientierungslosigkeit” und „Meinungspluralität” (fast alle Familienmitglieder haben ja eine andere politische Einstellung) stellt eine Analogie zur Situation im ganzen Europa dar.

\subsection{Andere Häuser}

Nicht nur die Behausung von den Ammans wird im Roman vorgestellt, sondern auch andere Häuser werden näher beschrieben. Von der Beschreibung des Hauses können wir auch auf die Persönlichkeit und auf die Beziehungen der Bewohner mit anderen Protagonisten schließen. Inglin schreibt grund- 
sätzlich wenig über den Schauplatz des Geschehens, aber die Wohnstätte werden in jedem Fall kurz beschrieben und sie verfügen über einen wichtigen Verweischarakter.

Ammans Schwager ist Divisionskommandant Boßhart. Das Haus, in dem Boßhart wohnt ist „ein nicht sehr geschmackvoller, aber solider eigenwilliger Bau mit einer Gartenterasse am Abhang" (S. 35). In diesem Haus hat Amman seine Frau kennengelernt und Gertrud ist ihrem Mann hier zum erstenmal begegnet. Man würde also denken, dass dieses Haus gute Gefühle erweckt. Trotzdem betritt er dieses Haus nur, wenn er es aus bestimmten Gründen muss. Wie die Beschreibung des Hauses zeigt, ist Boßhart ein eigenwilliger Mensch, dem das Ansehen sehr wichtig ist. Boßhart tritt in dieser Szene zu Amman ohne das geringste Zeichen von Wohlwollen, obwohl sie verwandt sind. (S. 36) Auch später, wenn sie miteinander reden, benutzt Boßhart einen scheinbar arglosen Ton, „aus dem aber Amman schon einen leisen Spott herauszuhören glaubte" (S. 246). Am Ende der Geschichte, wo Severin Boßhart wegen seinen Plänen besucht, redet er mit harter und klarer Stimme mit einer „sonderbaren, drohenden Schärfe” (S. 840), sodass Severin nicht wissen kann, ob er es ernst meint oder nur Scherz macht. Also wie die kurze Beschreibung des Hauses Vorbehalt im Leser weckt, so ist auch die ganze Persönlichkeit von Boßhart. Er ist eigenwillig und lässt niemanden in seine Nähe. Es wird wenig über Boßhart und sein Haus geschrieben, aber alles weist auf eine Überlegenheit und Zurückweisung hin.

Das Haus, wo Gertrud mit ihrem Mann lebt, wird als ein "noch ziemlich neuer, herrschaftlicher Bau in etwas undeutlichem Stil" (S. 44) beschrieben. Dort findet man auch einen kleinen Garten mit einem Seitenpfad zum Haupteingang. Das Haus ist noch ziemlich neu, die Bewohner noch jung und ihre Ehe ist auch relativ frisch. Weil sie zwei kleine Kinder haben, kann man darauf schließen, dass sie seit wenigen Jahren verhei- 
ratet sind. Und wie das Haus in einem undeutlichen Stil gebaut worden ist, ist auch die Art der Beziehung zwischen den zwei nicht ganz eindeutig definierbar bzw. die Persönlichkeit von Gertrud ändert sich mit der Zeit und durch das Geschehen. Die Eltern und Boßhart haben ihre Vorstellungen über das Leben und so werden ihre Häuser mit eindeutigen Worten beschrieben. Dagegen ändert sich Gertrud: wie sie die Welt sieht und was sie denkt, genauso hat das Haus einen undeutlichen Stil. Auch das Wort 'herrschaftlich' hat eine große Bedeutung. Hartmann ist ein eleganter Mann mit Haltung, Selbstsicherheit und Stolz. Erist ganz anders, als Gertruds neuer Geliebter, Albin.

Albin ist ein sehr empflindlicher Mensch, der dichtet und der unter dem Militärdienst sehr leidet, obwohl er nicht einmal kämpfen muss. Seine Beziehung mit Gertrud ist eher platonisch. Alles weist darauf hin, dass sie nur deswegen zusammengekommen sind, weil der Krieg ausgebrochen ist und sich alles verändert hat. Aber sie sind sehr unterschiedlich. Albin selber denkt, dass sich Gertrud an "ein geschertes, gepflegtes, auch im Alltäglichen kultiviertes Dasein gewöhnt” (S. 460) hat, was er ihr nicht bieten kann. Seine Wohnung ist in einem schmutziggrauen Haus, die Treppen sind schmal und schlecht beleuchtet, die Wohnung ist eng und dünnwandig, Albins Zimmer ist ein niedriger, ärmlicher Raum, der Fußboden knarrt und vom Treppenhaus kommt ältliche schlechte Luft in die Wohnung hinein. Das alles steht ganz im Gegenteil dazu, woran Gertrud sich gewöhnt hat, und auch wenn sie alle überzeugen will, dass das alles nicht zählt, muss Albin frisch rasiert und in seinem besten Anzug zu Gertrud gehen, wenn sie „es dort 'schön haben"' wollen (S. 566), weil Gertrud darauf Wert legt. Wie die Behausungen der zwei Männer völlig unterschiedlich sind, so unterscheiden sich auch die zwei Männer. Die Be- 
schreibung der Wohnstätte steht in Analogie zu ihren Bewohnern.

Wie man also sehen kann, ist nicht nur das Haus der Familie Amman wichtig, sondern auch die anderen Behausungen. Die Beschreibung der Wohnsitze weisen auf tiefere Zusammenhänge und Bedeutungen hin und ihre Wechsel verfügen über eine große Bedeutung im Lauf der Geschichte. Sie unterstützen und erklären im Hintergrund die Handlungen der Protagonisten, helfen beim Verstehen und symbolisieren Wertvorstellungen und Einstellungen.

\subsection{Das neue Haus}

Den Tiefpunkt erreicht die Familie, als Stockmeier ihnen die Miete kündigt, weil Amman seinen Sohn, Leo nicht auf geheimen Wegen in die Offizierschule zurücksetzte. Mit Schkanen vertreibt er die Familie schon vor dem Ablauf der Kündigungsfrist, wodurch sie gezwungen sind, kurzfristig in einem Hotel zu leben. Amman sagt Gertrud, dass sie in einem „Übergangsstadium" (S. 577) sind, als er mit ihr über die neue Situation redet. Er sagt, dass er genug von dem „Nomadenleben” (S. 578) hat. Dieser Ausdruck verweist sehr wohl nicht nur auf die Lebenssituation, sondern auch auf die Kopflosigkeit in der eigenen Familie. So kauft Amman ein Grundstück am Zürichberg.

Das neue Grundstück wurde die Zeit, wo Amman mit Gertrud redet schon gekauft und der Plan wurde bereits mit einem Architekten besprochen, aber bis das Haus aufgebaut wird, braucht man Zeit. Die Beziehungen sind ähnlicherweise am Tiefpunkt. Ammans „private [...] Verhältnisse waren ebenso gestört wie die öffentlichen" (S. 579). Amman überlegt, wem er überhaupt das Haus bauen sollte, weil die ganze Familie so zerrissen ist. Er kann sich nur noch Freds Rückkehr vorstellen, 
der gerade Militärdienst leistet. Wenn er an Paul denkt, wird er erbittert, aber auch auf Gertrud ist er böse, obwohl es ihm für Gertrud Leid tut. Und bald gerät seine Beziehung auch mit Severin in Krise, weil Severin die Zeitschrift alleine, ohne die Unterstützung seines Vaters weitermachen will, damit er seine politischen Gedanken weiterhin publizieren kann. "Jetzt war er [Amman] nirgend mehr zu Hause." (S. 560) Und das bezieht sich nicht nur auf die eigentliche Behausung, sondern auch auf sein Familien- und Privatleben.

Mann und Frau, beide erleben eine tiefe Erschütterung, wegen der auseinanderfallenden Familie. Barbara, die sich als Frau für den Zusammenhalt der Familie verantwortlich fühlt, kann diesen Stand nicht akzeptieren. Sie

sperrte sich im Innersten gegen die allgemeine Erschütterung, der sie entsprangen. [...] Was sie aber von der hintergründigen Strömung dieser Zeit, der elementaren Auflockerung des Lebens, nun hier persönlich an der eigenen Familie erfuhr, das wollte sie weder annehmen, noch begreifen. (S. 440f)

Amman fühlt sich „krank und müde” (S. 677) und ihm kommt es vor, als wenn nach dem Verkauf des alten Hauses eine Reihe böser Zufällle angefangen hätten, die ihn erbarmungslos treiben in ein bitteres Alter hinein, „das nicht mehr wert war, gelebt zu werden" (S. 677). Wie früher Barbara, macht er auch einen Rückblick auf sein Leben und denkt traurig, dass er statt eines Siegeskranzes für das tätige Leben nur Undank bekommt. Seine einzige Hoffnung ist, dass das Leben „im Ewigen begründet sei" (S. 679), obwohl er sich früher immer stolz von der Religiösität abgewendet hat.

Das neue Haus wird in Fluntern am Zürichberg gebaut. Amman kauft dort ein Grundstück mit schönem Blick auf die 
Stadt. Wenn ihm das Grundstück vor seinem Haus zum Kauf angeboten wird, denkt er, es sei nur Geldverschwendung ein Grundstück nur wegen dem Ausblick zu kaufen. Später bereut er jedoch seine Entscheidung. Sein neuer Nachbar wird Stockmeier sein, der ein großes Haus vor seinem bauen lässt. Das neue Verhältnis spiegelt tiefgreifende soziale Änderungen wider. Amman gehörte zum Bürgertum, das vor dem Weltkrieg eine leitende Position in der Gesellschaft hatte, aber nach dem Krieg verstärken sich andere gesellschaftliche Schichten. Die Lage der zwei Häuser zeigt die veränderten Machtverhältnisse auf eine sehr einfache, aber eindeutige Weise. Das ist beiden bewusst und Stockmeier genießt diese Situation besonders. Als sie auf der Straße zufällig zusammentreffen, sagt Stockmeier seinem Sohn laut genug, dass Amman und Fred das auch hört: „Mit den einen geht's hinauf, mit den andern hinab, das ist halt so im Leben, nicht wahr!” (S. 746). Diese Bemerkung ist Amman besonders schmerzhaft, weil sie ihm bewusst macht, was alles er in den letzten vier Jahren verloren hat.

Es ist wichtig zu erwähnen, dass das alte Haus, 1765 noch „mit freie[n] Gelände ringsum” (S. 19) angelegt wurde, aber mit der Zeit wurden geschmackslose Häuser um das elegante Herrenhaus gebaut, die auch den Ausblick blockiert haben. Genau diesen Prozess kann man bei dem neuen Haus beobachten, mit dem einzigen Unterschied, dass das hier viel schneller passiert.

Über das neue Haus wird wenig berichtet. Schon das hat eine sinntragende Bedeutung. Das alte Haus war mit seiner Pracht detailliert beschrieben, was darauf hinweist, dass das Leben damals auch ein reiches und stolzes Leben war. Das neue Leben, das Leben nach dem Krieg ist nur wie Schatten der damaligen Zeit. Noch dazu wird das neue Haus zum ersten Mal nach dem Begräbnis eines Familienmitgleids vorgestellt. Alleine das weist auf die Auflösung von etwas hin. Das neue Haus 
„wirkt behäbig” (S. 668), ist zweistöckig und seine einfachen Formen erinnern an „das verkaufte und verschwundene Herrschaftshaus” (S. 668). Jedoch gab es beim neuen Haus „ein[en] schmale[n] Gartenstreifen, der noch dürftig war" (S. 668). Bei dem alten Haus gab es Hecke, alte Parkbäume, Sandsteinfliesen über einen Rasenstreifen. Alles was zu einem eleganten Haus gehört. Im neuen Haus gab es nur ein bescheidenes Vestibül, im alten Haus gab es eine Vorhalle. Das zeigt, wie die Familie versucht, die alten Verhältnisse wiederherzustellen, aber das gelingt ihnen nur teilweise. Das neue Haus kann an das alte bloß erinnern, es wird aber nie so prachtvoll. Genauso ist es auch im Familienleben. Die Beziehungen können teilweise wiederhergestellt werden, aber nichts wird das Alte sein. Das Innere des Gebäudeswird nur kurz erwähnt. Es wird jedoch betont, dass im Haus viele Zimmer zu finden sind. Severins Frau ist verwundert, als sie das Haus sieht und sagt: „Man würde nicht glauben, daß in diesem Hause soviel Platz ist” (S. 675f) Es wird nicht begründet, warum so viele Zimmer gebaut wurden, aber man kann darauf schließen, dass die Eltern doch auf die Rückkehr der Kinder hoffen. Das ist hier genau das Gegenteil davon, was man über die Miete bei Stockmeier erfahren hat, wo ein Zimmer zu wenig war. Und wie dort die wenigen Zimmer auf den Zerfall der Familie hinwiesen, so weisen hier die vielen Zimmer darauf, dass die Familie sich wieder zusammenstellt. Was mit der Zeit tatsächlich geschieht. Barbara holt Paul, der mit der spanischen Grippe angesteckt wird, vom Krankenhaus heim und vermittelt zwischen Vater und Sohn. So werden sie die Meinung des anderen akzeptieren, auch wenn sie nicht damit einverstanden sind. Nachdem Albin gestorben ist, lädt Barbara Gertrud ein, um ihr um die Kranken im Haus zu helfen. Obwohl Gertrud nicht heimkehren will, willigt sie doch ein und kann sich mit der Zeit mit ihrer Mutter versöhnen. 
Auch die Beziehungen zwischen den französisch- und deutschsprachigen Familienmitgliedern verbessern sich. Nach dem Tod von Ammans Schwester ist die ganze Familie in das neue Haus eingeladen. Hier erscheinen Paul und Gertrud noch nicht, obwohl Barbara sie auch in der Hoffnung auf eine Versöhnung eingeladen hat, aber die Welsch-Schweizer versöhnen sich mit den Ammans. Das neue Haus wird zu einem Ort der Versöhnung und steht für die Hoffnung auf ein friedlicheres Leben.

Am Ende der Geschichte kehrt der Frieden in die Familie zurück. Doch sind die Wunden immer noch da. Das neue Haus ist nicht so prachtvoll und heimisch, wie das alte war, genau so ist die Familie nicht mehr die alte. Obwohl die Familienmitglieder wieder miteinander reden, müssen sie aufpassen, was und wie sie sagen. Auch ihr sozialer Stand ist nicht mehr so bedeutend, wie er vor dem Krieg war. Damals hat sich Stockmeier vor ihnen gebeugt, jetzt spottet er über sie laut auf der Straße.

Der Frieden kehrt aber nicht nur in die Familie zurück. Auch der Krieg ist zu Ende. Wenn man die großen Katastrophen in Europa betrachtet, kann man fragen, wo die Katastrophe der Schweiz ist, oder wo die Tragödie in diesem Roman ist. Scheinbar lösen sich die Probleme und mit wenigen Toten geht das Leben weiter, aber in der Wirklichkeit haben die Menschen ihre unerschütterliche Zuversicht verloren. Auch im Roman merkt der Leser, dass die Familie nicht weiß, wie das Leben weitergeht. Die Ammans haben ihren festen Stand verloren, freuen sich aber zugleich, dass sie überhaupt ein eigenes $\mathrm{Zu}$ hause haben und, dass sie weiterhin eine Familie sind. Diese Art Tragödie ist die Tragödie der Schweiz. Das Land hat nicht Tausende von Bürgern verloren, aber der Krieg ist auch hier nicht spurlos vergangen. Dieser Roman arbeitet den Krieg aus 
seiner Perspektive auf und erzählt über eine andere Art von Tragödie, als die anderen Werke über den Ersten Weltkrieg.

\section{Zusammenfassung}

Die vorliegende Arbeit beschäftigt sich mit dem berühmtesten Roman von dem schweizerischen Autor Meinrad Inglin, mit dem Schweizerspiegel. Das Ziel der Arbeit war die Rolle der Behausungen im Roman zu untersuchen und Parallelen zwischen dem jeweiligen Wohnsitz und dem Innenleben der Familie zu finden, bzw. die Analogie zwischen der Behausung und dem Bewohner nachzuweisen. Meine Hypothese war, dass der jeweilige Wohnort das Innenleben, die Einstellung und Wertvorstellung der Protagonisten widerspiegelt.

Die drei Wohnorte, wo die Familie im Roman wohnt, sind im Werk von großer Wichtigkeit. Das wird schon dadurch bestätigt, dass es im ganzen ersten Kapitel nur um den Verkauf des alten Hauses geht; darüber hinaus werden die Schauplätze im Text nicht ausführlich beschrieben, aber die Wohnorte werden detaillierter vorgestellt. Der erste Wohnsitz, das alte Haus steht für die bürgerlichen Verhältnisse und für den familiären Frieden. Mit dem Verkauf des Hauses werden diese Verhältnisse und dieser Frieden zerstört, die neue Wohnung bietet weder den früher gewohnten Luxus noch den gewünschten Frieden für die Familie. Dieser Zeit bricht auch der Erste Weltkrieg aus, der die privaten Konflikte mit politischen und gesellschaftlichen Problemen steigert. Am Ende des Romans, wo der Krieg langsam zu Ende ist, wird ein neues Haus gebaut, und auch die Konflikte werden innerhalb der Familie teilweise gelöst. Durch den Wechsel der Häuser ist das Familienleben gut beobachtbar und interpretierbar. Dadurch ist auch meine Hypothese bestätigt, nach der die Wohnsitze der Familie tatsäch- 
lich die Beziehungen und die Konflikte zwischen den Protagonisten widerspiegeln.

Jedoch bleibt am Ende die Frage offen, wie das Familienleben nach dieser krisenhaften Zeit weitergeht. Es wird darauf verwiesen, dass die Kinder teilweise heimkehren; aber das, was sie erlebt haben, hat sie verändert. Der Roman gibt keine eindeutige Antwort darauf, in wie weit das das Leben der Familie im Folgenden beeinflusst. Wie es auch keine Antwort im Schweizerspiegel auf die politisch-ideologischen Fragen des Romans zu finden ist.

Ich habe kurz auch andere Wohnstätte untersucht und ich bin darauf gekommen, dass auch andere Behausungen im Roman die Bewohner sehr stark charakterisieren. Das zeigt, dass Inglin den Schauplatz des Geschehens dafür verwendet hat, die Ereignisse und Charaktere aus einer anderen Sicht zu beleuchten und dadurch zu einem besseren Verständnis zu verhelfen. Hier lässt sich gut verfolgen, wie der Raum in literarischen Werken die Handlung und die Figurenkonstellation übersichtlicher und nachvollziehbarer macht. Das heißt, durch die Analyse der Räume, bzw. ganz spezifisch durch die Analyse der Behausungen kommt man näher zum Verständnis der komplexen Handlung und zur Struktur des Textes.

Dieser Roman ist wenig bekannt, so wurde er nicht oft untersucht, obwohl er zahlreiche andere Themen bietet, die untersuchungswert wären. Wie ich früher schon erwähnt habe, die Rolle des „Vorspiels” wäre interessant zu untersuchen, wie auch die Frage, inwieweit der Roman das kulturelle Gedächtnis über den Ersten Weltkrieg in der Schweiz beeinflusste. Es wäre natürlich auch möglich, einen Vergleich mit anderen Familienromanen, wie z. B. mit den Buddenbrooks zu machen, oder die Darstellung der geschichtlichen Ereignisse, sowie ihre Rolle im Roman zu untersuchen. 
Der Schweizerspiegel ist ein vielversprechendes Buch für zahlreiche Untersuchungaspekte. Mit meiner Arbeit versuchte ich es zu beweisen, dass er größerer literarischer Beachtung wert wäre. Weiterhin zielte ich darauf, dass das Buch bekannter wird und hoffentlich das Interesse erweckt.

\section{Literaturverzeichnis}

\section{Primärliteratur}

Inglin, Meinrad (2014): Schweizerspiegel. Zürich: Limmat Verlag.

\section{Sekundärliteratur}

Aczél, Judit (Hrsg.) (1997): Ne félj! Beszélgetések Szabó Magdával. Debrecen: Csokonai Kiadó.

Baumgärtner, Ingrid/Klumbies, Paul-Gerhard/Sick, Franziska (Hrsg.) (2009): Raumkonzepte. Göttingen: V\&R Unipress.

Bollnow, Otto Friedrich (1962): Der bergende Raum. In: Duitse Kroniek, 14. Jg. 1962, S. 49-62.

Bollnow, Otto Friedrich (1979): Der Raum. In: Ders.: Die Ehrfurcht vor dem Leben. Erläutert von K. Suzuki. Tokyo: Asahi Verlag, S. 15-22.

Brynhildvoll, Knut (1993): Der literarische Raum. Konzeptionen und Entwürfe. Berlin: Peter Lang. 
Dennerlein, Katrin (2011): Raum. In: Martínez, Matías (Hrsg.): Handbuch. Erzählliteratur. Stuttgart/Weimar: Metzler, S. 158-165.

Döring, Jörg/ Thielmann, Tristan (2008): Einleitung Was lesen wir im Raume? Der Spatial Turn und das geheime Wissen der Geographen. In: Döring, Jörg / Thielmann, Tristan (Hrsg.) (2008): Spatial Turn: Das Raumparadigma in den Kultur- und Sozialwissenschaften Bielefeld: Transcript. S. 7-45.

Egger, Hartmut/Golec, Janusz (Hrsg.) (2004): Lügen und ihre Widersacher: Literarische Ästhetik der Lüge seit dem 18. Jahrhundert. Würzburg: Königshausen \& Neumann.

Foucault, Michel (1992): Andere Räume. In: Barck, Karlheinz u.a. (Hrsg.), Aisthesis. Wahrnehmung heute oder Perspektiven einer anderen Ästhetik. Leipzig: Reclam, S. 34-46.

http://www.containerwelt.info/pdf/Foucault_AndereRaeume.pdf (zuletzt gesehen am 24.07.2018)

Grimm, Jacob/Grimm, Wilhelm (1984): Deutsches Wörterbuch von Jacob und Wilhelm Grimm. Band 14. München: Deutscher Taschenbuch Verlag.

Hallet, Wolfgang/Neumann, Birgit (Hrsg.) (2009): Raum und Bewegung in der Literatur: Die Literaturwissenschaften und der Spatial Turn. Bielefeld: Transcript.

Inglin, Meinrad/Hangarten, Felix R. (Hrsg.) (1992): Die Briefwechsel mit Traugott Vogel und Emil Staiger. Zürich: Amman.

Inglin, Meinrad/Schoeck-Grüebler, Elisabeth (Hrsg.) (2005): Seine Welt in Bildern. Zürich: Limmat. 
Innerhofer, Roland (2012): Literatur und Architektur in der Moderne. In: IGGerm.

http://www.univie.ac.at/iggerm/files/mitschriften/sose12/Archi tektur_und_Literatur2-SS12-Innerhofer.pdf (zuletzt gesehen am 24. 11.2017)

Martínez, Matías (Hrsg.) (2011): Handbuch. Erzählliteratur. Stuttgart/Weimar: Metzler.

Matt, Beatrice von (1976): Meinrad Inglin. Eine Biographie. Zürich:_Amman.

Matt, von Beatrice (2014): Nachwort. In: Inglin, Meinrad: Schweizerspiegel. Zürich: Limmat. S. 875-900.

Nünning, Ansgar (2009): Formen und Funktionen literarischer Raumdarstellung: Grundlagen, Ansätze, narratologische Kategorien und neue Perspektiven. In: Hallet, Wolfgang/Neumann, Birgit (Hrsg.): Raum und Bewegung in der Literatur: Die Literaturwissenschaften und der Spatial Turn. Bielefeld: Transcript. S. 33-52.

Piatti, Barbara (2008): Die Geographie der Literatur: Schauplätze, Handlungsräume, Raumphantasien. Göttingen: Wallstein.

Ritter, Rüdiger (2004): Wem gehört Musik? Warschau und Wilna im Widerstreit nationaler und städtischer Musikkulturen vor 1939. Stuttgart: Franz Steiner.

Rusterholz, Peter/Solbach, Andreas (Hrsg.) (2007): Schweizer Literaturgeschichte. Stuttgart: J. B. Metzler. 
Schlögel, Karl (2003): Im Raume lesen wir die Zeit. München: Carl Hanser Verlag.

Schneider-Handschin, Esther (1997): Kakanien im Schweizerhaus? - Zu Robert Musils Mann ohne Eigenschaften und Meinrad Inglins Schweizerspiegel. In: Modern Austrian Literatur 30, H. 3/4, S. 144-157.

Schroer, Markus (2008): „Bringing space back in”- Zur Relevanz des Raums als soziologischer Kategorie. In: Döring, Jörg/Thielmann, Tristan (Hrsg.): Spatial Turn: Das Raumparadigma in den Kultur- und Sozialwissenschaften. Bielefeld: Transcript, S. 125-148.

Würzbach, Natascha (2001): Erzählter Raum. Fiktionaler Baustein, kultureller Sinnträger, Ausdruck der Geschlechtsordnung. In: Helbig, Jörg (Hrsg.): Erzählen und Erzähltheorie im 20. Jahrhundert. Festschrift für Wilhelm Füger, Heidelberg: Winter, S. 105-129. 